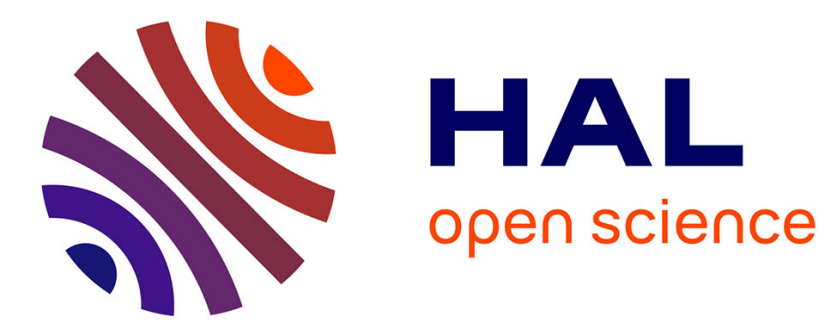

\title{
Création d'une matrice emplois-expositions sur le travail à horaires atypiques
}

\author{
Vy Sok, Nastassia Tvardik, Emilie Cordina, Emilie Pelletan, Marie Houot, \\ Corinne Pilorget, Pascal Guenel
}

\section{- To cite this version:}

Vy Sok, Nastassia Tvardik, Emilie Cordina, Emilie Pelletan, Marie Houot, et al.. Création d'une matrice emplois-expositions sur le travail à horaires atypiques. 35e congrès national de médecine et santé au travail, Jun 2018, MARSEILLE, France. p. 400, 10.1016/j.admp.2018.03.427 . hal02266149

\section{HAL Id: hal-02266149 \\ https://hal.science/hal-02266149}

Submitted on 13 Aug 2019

HAL is a multi-disciplinary open access archive for the deposit and dissemination of scientific research documents, whether they are published or not. The documents may come from teaching and research institutions in France or abroad, or from public or private research centers.
L'archive ouverte pluridisciplinaire HAL, est destinée au dépôt et à la diffusion de documents scientifiques de niveau recherche, publiés ou non, émanant des établissements d'enseignement et de recherche français ou étrangers, des laboratoires publics ou privés. 
- Depuis plusieurs années, le travail à horaires atypiques, et le travail de nuit en particulier, suscite de fortes inquiétudes liées à ses effets avérés ou supposés sur la santé. En 2007, le Centre international de recherche sur le cancer (CIRC) classait le travail posté comme probablement cancérogène pour l'homme (2A) par son rôle de perturbateur du rythme circadien. Dans ce contexte, nous avons souhaité développer un outil permettant une évaluation exhaustive et rétrospective des emplois à horaires atypiques en distinguant les différents types d’horaires ainsi que son évolution au cours du temps. La construction de la matrice est basée sur les données des Enquêtes emploi (EE) de l’Institut national des statistiques et des études économiques (INSEE). Ces enquêtes transversales répétées au cours du temps, contiennent des informations sur l'emploi et les conditions de travail recueillies au niveau individuel sur un échantillon représentatif de la population française en métropole et dans les DOM. La matrice, basée sur les professions et les branches d'activités, est construite à partir d'une méthode de segmentation des données, dite méthode d'inférence conditionnelle. Les contraintes organisationnelles évaluées dans la matrice sont l'existence d'un travail du soir entre 20 heures et minuit (régulier, occasionnel, jamais), d'un travail de nuit entre minuit et 5 heures (régulier, occasionnel, jamais), ou d'un travail à alternance régulière ou irrégulière. Cette matrice indique la probabilité de travailler de soir, de nuit ou d'avoir un travail posté en fonction de la profession, du secteur d'activité en France et de l'année de l'emploi. Elle permettra l'évaluation et la mesure de la perturbation du rythme circadien en milieu de travail ainsi que l'étude des liens entre le travail à horaires décalés et la santé. 